\title{
Amygdala Inputs to the Ventral Hippocampus Bidirectionally Modulate Social Behavior
}

\author{
Ada C. Felix-Ortiz and Kay M. Tye \\ Picower Institute for Learning and Memory, Department of Brain and Cognitive Sciences, Massachusetts Institute of Technology, Cambridge, \\ Massachusetts 02139
}

Impairments in social interaction represent a core symptom of a number of psychiatric disease states, including autism, schizophrenia, depression, and anxiety. Although the amygdala has long been linked to social interaction, little is known about the functional role of connections between the amygdala and downstream regions in noncompetitive social behavior. In the present study, we used optogenetic and pharmacological tools in mice to study the role of projections from the basolateral complex of the amygdala (BLA) to the ventral hippocampus (vHPC) in two social interaction tests: the resident-juvenile-intruder home-cage test and the three chamber sociability test. BLA pyramidal neurons were transduced using adeno-associated viral vectors $\left(\mathrm{AAV}_{5}\right)$ carrying either channelrhodopsin-2 (ChR2) or halorhodopsin (NpHR), under the control of the CaMKII $\alpha$ promoter to allow for optical excitation or inhibition of amygdala axon terminals. Optical fibers were chronically implanted to selectively manipulate BLA terminals in the vHPC. NpHR-mediated inhibition of BLA-vHPC projections significantly increased social interaction in the resident-juvenile intruder home-cage test as shown by increased intruder exploration. In contrast, ChR2-mediated activation of BLA-vHPC projections significantly reduced social behaviors as shown in the resident-juvenile intruder procedure as seen by decreased time exploring the intruder and in the three chamber sociability test by decreased time spent in the social zone. These results indicate that BLA inputs to the vHPC are capable of modulating social behaviors in a bidirectional manner.

Key words: amygdala; ChR2; hippocampus; NpHR; optogenetics; social

\section{Introduction}

Major psychiatric disorders, such as depression, autism, schizophrenia, and social anxiety disorder, share impaired social interaction as a distinctive feature (American Psychiatric Association, 2013). However, little is known about the neural circuitry regulating adult social interaction. Previous studies using pharmacological interventions or lesions have suggested an involvement of the amygdala in social behaviors and social aggression in both nonhuman primates (Kling, 1974; Kling and Steklis, 1976; Machado et al., 2008) and rodents (Bunnell et al., 1970; Jonason and Enloe, 1971; Sanders and Shekhar, 1995a, b; Gonzalez et al., 1996). In vivo electrophysiology recordings revealed changes in neuronal activity in the basolateral amygdala (BLA) during social interaction behaviors, such as increased firing in the BLA related

\footnotetext{
Received Oct. 3, 2013; revised Nov. 21, 2013; accepted Nov. 24, 2013.

Author contributions: A.C.F.O. and K.M.T. designed research; A.C.F.O. performed research; A.C.F.O. analyzed data; A.C.F.O. and K.M.T. wrote the paper.

This work was supported by the Whitehall Foundation, Klingenstein Foundation, Picower Institute Innovation Funds, Whitehead Career Development Professorship, and the New Innovator Award (1DP2DK102256-01). We thank Anthony Burgos-Robles, Neha D. Bhagat, and Romy Wichmann for technical advice; Gillian A. Matthews, a Simons Center for the Social Brain postdoctoral fellow, for useful comments which have greatly improved the manuscript; and the entire Tye laboratory for helpful discussion.

The authors declare no competing financial interests.

Correspondence should be addressed to Dr. Kay M. Tye, Picower Institute for Learning and Memory, Department of Brain and Cognitive Sciences, Massachusetts Institute of Technology, 77 Massachusetts Avenue, Building room 46-6263, Cambridge, Massachusetts 02139. E-mail: kaytye@mit.edu.

DOI:10.1523/JNEUROSCI.4257-13.2014

Copyright $\odot 2014$ the authors $\quad 0270-6474 / 14 / 340586-10 \$ 15.00 / 0$
}

to the augmentation of general social behavior (Katayama et al., 2009). However, the way the amygdala interacts with other downstream regions in a social context is poorly understood.

One downstream region of the amygdala that has been implicated in social interaction in rodents is the ventral hippocampus (vHPC), which shares reciprocal connections with the BLA (O'Donnell and Grace, 1995; Pikkarainen et al., 1999). Lesion studies provided the first evidence of an involvement of the vHPC in social interaction (Cadogan et al., 1994; Deacon et al., 2002; McHugh et al., 2004) and defensive behaviors (Pentkowski et al., 2006). Furthermore, studies using selective lesion methods have suggested that the vHPC, but not the dorsal hippocampus, is required for social interaction during a resident-intruder test (McHugh et al., 2004). Despite these previous findings, it remains unclear whether the BLA interacts with the vHPC to modify social behavior.

Our recent work demonstrated that the activation of BLA projections to the central nucleus of the amygdala (Tye et al., 2011) or the vHPC (Felix-Ortiz et al., 2013) mediates opposing effects on anxiety-related behaviors. However, the role of specific BLA projections in social behavior has not previously been explored. To selectively control projections from the BLA to specific downstream targets, we took advantage of the cellular specificity and temporal precision of optogenetics. This allowed neural activation or inhibition on a timescale relevant to social approach in two well-validated behavioral procedures (residentintruder and the three chamber sociability test) (File and Pope, 
A

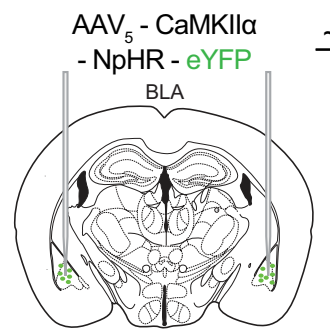

$\stackrel{\sim 7 \text { weeks }}{\longrightarrow}$

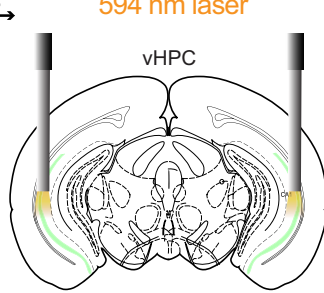

B
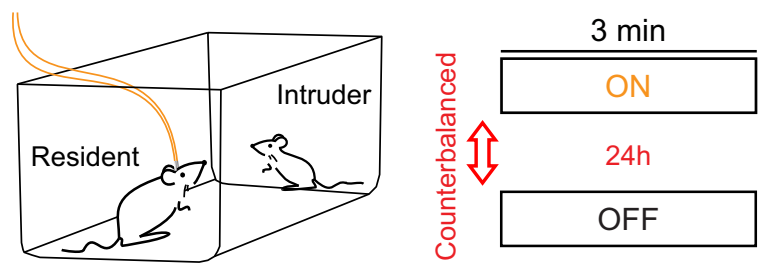

C NpHR:BLA-vHPC $(n=7)$

eYFP:BLA-vHPC $(n=8)$

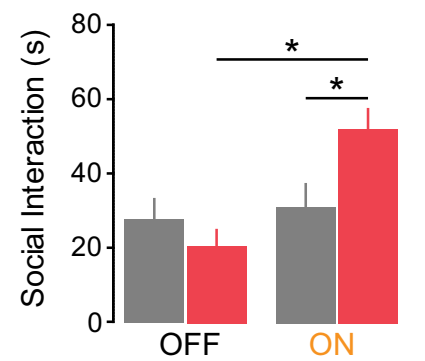

E

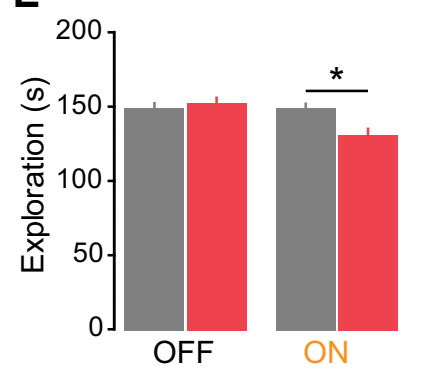

D

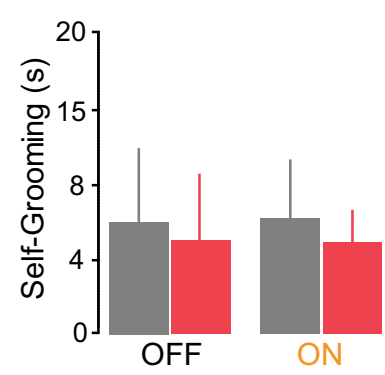

$\mathbf{F}$
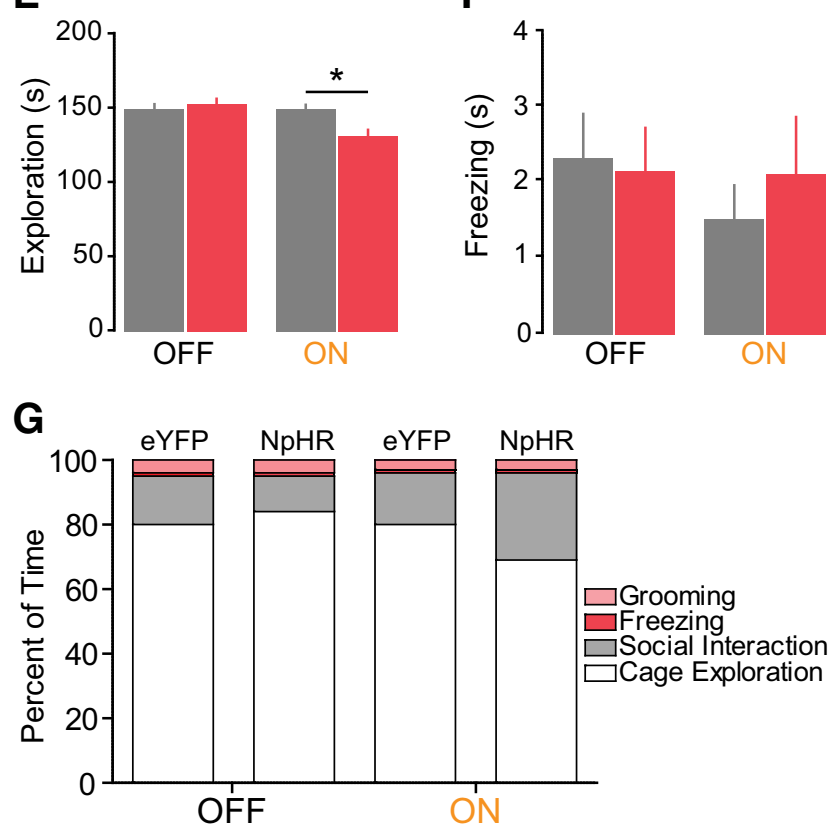

Figure 1. Inhibition of BLA terminals projecting to the VHPC with NpHR increases social interaction in a resident-juvenile intruder procedure. BLA glutamatergic neurons were transduced with either NpHR-eYFP $(n=7)$ or eYFP alone as a control $(n=8)$. Yellow light was delivered ( 3 min, constant) via bilateral optical fibers implanted in the vHPC, after $\sim 7-8$ weeks of viral incubation. $A$, Left, Coronal brain schematic indicating the site of viral injection into the BLA. Right, Coronal schematic indicating optic fiber location into the VHPC. Top, Experimental timeline. $\boldsymbol{B}$, Left, Schematic indicating the home-cage resident-juvenile (3-4 weeks) intruder behavioral procedure. Right, Schematic indicating the 3 min epochs with $24 \mathrm{~h}$, counterbalanced between the social tasks. Different intruders were used for each epoch. $C$, NpHR mice spent significantly more time performing social interaction than eYFP mice during the yellow light
1974; Winslow, 2003; Silverman et al., 2010; Kaidanovich-Beilin et al., 2011; Himmler et al., 2013). These findings illustrate an approach based on optogenetic tools for testing the causal significance of circuits characterized by cell location, cell type, projection target, and target receptors in freely moving mammals executing complex behaviors.

\section{Materials and Methods}

Animals

Naive wild-type male C57BL/6 mice, 5-6 weeks of age (The Jackson Laboratory) were group-housed in clear Plexiglas cages with ad libitum access to food and water until surgery. After surgery, mice were maintained on a $12 \mathrm{~h} \mathrm{light/dark} \mathrm{cycle.} \mathrm{The} \mathrm{stimulus} \mathrm{(juvenile} \mathrm{intruder)} \mathrm{mice}$ were 3- to 4-week-old male C57BL/6 mice (The Jackson Laboratory), group housed. All animal procedures were approved by the Massachusetts Institute of Technology Institutional Animal Care and Use Committee and conducted in accordance with the NIH Guide for the Care and Use of Laboratory Animals.

\section{Surgery}

Mice were anesthetized with $1.5-2.0 \%$ isoflurane gas/oxygen mixture and aligned on the stereotaxic apparatus (Kopf Instruments). Body temperature was maintained throughout surgery with an adjustable warming pad. All stereotaxic coordinates were described relative to bregma. Surgery was performed for viral transduction of BLA cell somata $(-1.16$ $\mathrm{mm}$ anteroposterior; $\pm 3.3-3.4 \mathrm{~mm}$ mediolateral; and $-4.9 \mathrm{~mm}$ dorsoventral). A midline incision was made down the scalp, and craniotomy was made using a dental drill. A $10 \mu \mathrm{l}$ microsyringe (nanofil; WPI) was used to deliver virus solution at a rate of $0.1 \mu \mathrm{l} / \mathrm{min}$ using a microsyringe pump (UMP3; WPI) and its controller (Micro4; WPI). The virus solution contained purified adeno-associated viral vector serotype $5\left(\mathrm{AAV}_{5}\right)$, under the control of a CaMKll $\alpha$ promoter, coding eNpHR3.0 fused to an enhanced yellow fluorescent protein (eNpHR3.0-eYFP), or channelrhodopsin-2 (ChR2)(H134R)-eYFP, or eYFP alone. $\mathrm{AAV}_{5^{-}}$ CaMKII $\alpha$-eNpHR3.0-eYFP or eYFP was injected bilaterally $(0.5 \mu \mathrm{l}$ per side) for mice in Figure $1 . \mathrm{AAV}_{5}$-CaMKII $\alpha$-ChR2(H134R)-eYFP or eYFP was injected unilaterally $(0.5 \mu \mathrm{l})$ for mice in Figures 2, 3, and 4. All virus aliquots were obtained from the University of North Carolina Vector Core (Chapel Hill, NC). The maps for above constructs are available online at www.optogenetics.org.

After infusion, the needle was kept at the injection site for $10 \mathrm{~min}$ and then slowly withdrawn. To allow for projection-specific targeting of BLA terminals, mice were implanted bilaterally or unilaterally with chronic implantable optic fibers (length $=4 \mathrm{~mm}, 300 \mu \mathrm{m}$ core, $\mathrm{NA}=0.37$; Thorlabs) held in a stainless steel ferrule (Precision Fiber Products) over the vHPC $(-3.08 \mathrm{~mm}$ anteroposterior; $\pm 3.6-3.7 \mathrm{~mm}$ mediolateral; $-3.4 \mathrm{~mm}$ dorsoventral). For pharmacological experiments, a unilateral cannula (22GA, PlasticsOne) was implanted above the vHPC for infusion of glutamate receptor antagonists. Implanted fibers and cannulae were adhered to the skull with a layer of adhesive cement (C\&B Metabond; Parkell) followed by dental cement (Ortho-Jet; Lang). The cement was allowed to dry before the incision was closed using nylon sutures, and the animal was then allowed to recover from anesthesia under a heat lamp to maintain body temperature. Mice were given a 6 to 7 week incubation period to allow for proper viral transduction to the vHPC terminals, before behavioral experiments.

\section{Laser delivery}

A 3-m-long fiber-optic patch cord (Doric) was connected to the chronically implanted optic fiber and suspended above the behavioral

$\leftarrow$

illumination epoch. ${ }^{*} p=0.046$. $\boldsymbol{D}$, No significant effect of light stimulation or group was detected on time spent self-grooming compared with eYFP mice. $\boldsymbol{E}$, NpHR mice spent less time (seconds) exploring their home cage during light stimulation compared with eYFP control mice. ${ }^{*} p=0.035$. $\boldsymbol{F}$, No significant effect of light stimulation or group was detected on freezing behavior in the presence of a juvenile intruder. $\mathbf{G}$, Percentage of total time (3 min), showing social interaction, self-grooming, cage exploration, and freezing. Data are mean values. Error bars indicate SEM. 
testing arena to allow animals to interact and move freely while receiving laser stimulation. The patch cord was connected to a 594 $\mathrm{nm}$ laser or a $473 \mathrm{~nm}$ laser (OEM Laser Systems) with an FC/PC adapter. All laser output was manipulated with a Master- 8 pulse stimulator (A.M.P.I.). For mice in Figure 1 (bilateral optogenetic inhibition of BLAvHPC projection using NpHR3.0), bilateral inhibition through two implanted chronic optic fibers connected to the optic patch cords (Doric) received $10 \mathrm{~mW}(\sim 35.35 \mathrm{~mW} /$ $\mathrm{mm}^{2}$ ) of constant yellow light generated by a $594 \mathrm{~nm}$ DPSS Laser (OEM Laser Systems). For mice in Figures 2, 3, and 4, unilateral activation using ChR2(H134R) was achieved by delivering $10 \mathrm{~mW}\left(\sim 35.35 \mathrm{~mW} / \mathrm{mm}^{2}\right)$ of blue light in a high-frequency train $(20 \mathrm{~Hz}, 5$ ms pulses) generated by a $473 \mathrm{~nm}$ DPSS laser (OEM Laser Systems).

\section{Behavioral assays}

All tests were performed during the dark phase, and animals were allowed to acclimate to the behavioral testing room for at least $1 \mathrm{~h}$ before the beginning of testing.

\section{Social interaction assay}

Male juvenile mice were used instead of adults to exclude any effect of mutual aggression. Social interaction in the home cage was examined as described previously (Winslow, 2003). A single mouse was allowed to explore freely for 1 min (habituation) in his home cage. A novel juvenile (3-4 weeks old) male C57BL/6 mouse was introduced to the cage and allowed to explore freely for $3 \mathrm{~min}$ (test session). All behaviors were video recorded and analyzed by an experimenter blind to the testing condition using ODLog software (Macropod software). The overall score of social interaction included behaviors, such as body sniffing, anogenital sniffing, direct contact (pushing the snout or head underneath the juvenile's body and crawling over or under the juvenile's body), and close following $(<1 \mathrm{~cm})$. Nonsocial behaviors were also represented in an overall exploration score, which included walking, rearing, freezing, and self-grooming. Each mouse underwent two social interaction tests separated by $24 \mathrm{~h}$, with one intruder paired with optical stimulation and one with no stimulation. Groups were counterbalanced for order of light stimulation.
A Viral injection in BLA:

- $\mathrm{AAV}_{5}$-CaMKIla-NpHR-eYFP $(n=7)$

- $\mathrm{AAV}_{5}$-CaMKIla-eYFP $(n=8)$
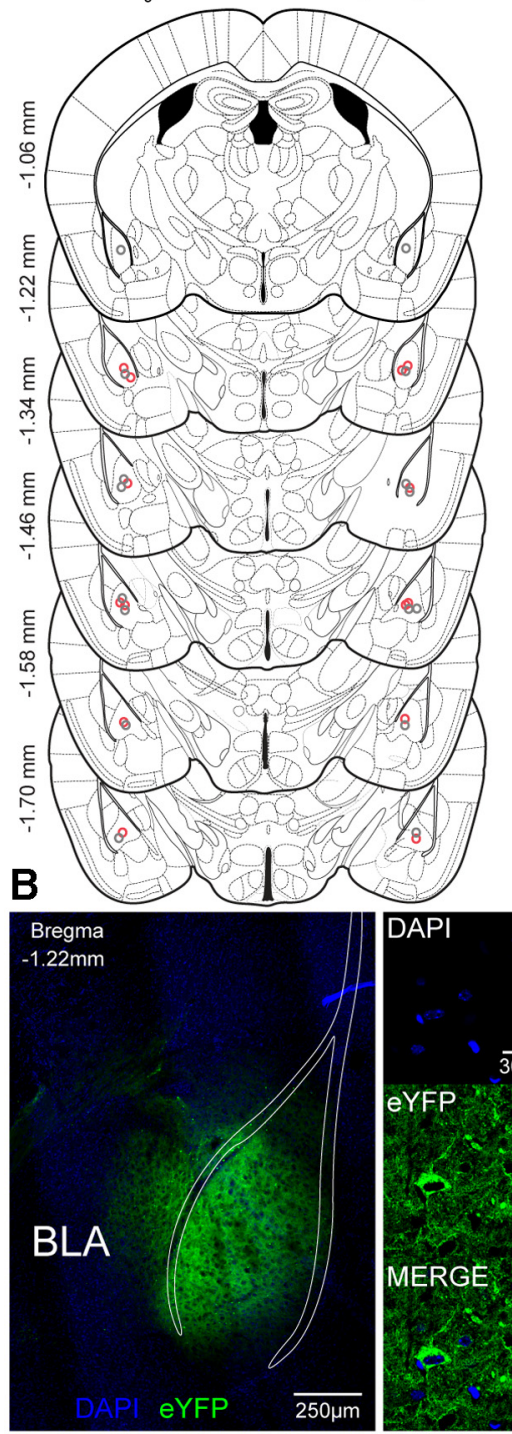

DAPI

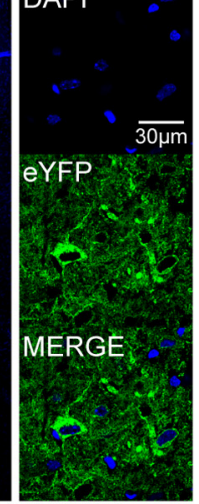

Figure 2. Histologically verified placements of viral injections and optical fiber tips in NpHR:BLA-vHPC and eYFP:BLA-vHPC groups. $A$, Coronal sections from bregma containing the BLA. Center of the viral injections in the BLA for all the mice injected with $\operatorname{NpHR}(n=7$; orange circles) and eYFP ( $n=8$; gray circles). $\boldsymbol{B}$, BLA confocal image from a representative NpHR mouse. Right, Confocal images of individual cells in the BLA of a mouse in the NpHR group. $C$, Coronal sections from bregma containing the vHPC. Location of the optical fiber tips above the pyramidal layer of vHPC for NpHR:BLA-vHPC (orange crosses) and eYFP (gray crosses). D, vHPC confocal image indicating the optical fiber placement in a representative mouse from the NpHR group. Right, Confocal images of individual cells in the vHPC of a mouse expressing NpHR in BLA axon terminals.

\section{Three chamber sociability test}

Apparatus. A different cohort of animals was subjected to the three chamber sociability test (Fig. 4). The three compartment testing apparatus consisted of a Plexiglas rectangular box $(57.15 \mathrm{~cm} \times 22.5 \mathrm{~cm} \times 30.5 \mathrm{~cm})$, without a top. The center compartment was smaller $(8 \mathrm{~cm} \times 22.5 \mathrm{~cm})$ than the two end compartments, which were of equal size $(24.5 \mathrm{~cm} \times$ $22.5 \mathrm{~cm}$ ). Inverted custom made wire cups (diameter $8 \mathrm{~cm}$ ) were placed in each side of the end compartments during testing sessions (discussed below) and housed the juvenile stimulus mouse. The apparatus and wire cups were thoroughly cleaned with $70 \%$ ethanol between sessions and after each test mouse.

Sociability procedure. In the first session, a test mouse was placed in the middle compartment and allowed to habituate to the apparatus for 10 $\mathrm{min}$, and the first $5 \mathrm{~min}$ was used for analysis. In the second $5 \mathrm{~min}$ session, a stimulus juvenile (3-4 weeks) mouse was placed in an inverted wire cup in the side designated as the social compartment, and an empty inverted wire cup was placed in the side designated as the nonsocial compartment. The test mouse was placed in the middle chamber for 1-2 min while the experimenter placed the juvenile mouse inside the inverted cup. The test mouse was then left to explore both chambers for $5 \mathrm{~min}$. The side designated for the location of the enclosed stimulus mouse was randomly assigned in a counterbalanced fashion throughout the $2 \mathrm{~d}$ experiment. Behavioral tests were recorded by a video camera, and the EthoVision XT video tracking system (Noldus) was used to track mouse location, velocity, and movement of head, body, and tail. All measurements were made relative to the mouse body. The amount of time test mice spent in each compartment, the amount of time they explored (sniffing) within a $2 \mathrm{~cm}$ vicinity of the inverted cups, and their transitions between compartments were measured. Each mouse underwent the above sociability task twice, separated by $24 \mathrm{~h}$, with one session paired with optical stimulation 
and one with no stimulation. Groups were counterbalanced for order of light stimulation as well as side assigned as the social zone.

\section{Pharmacology}

A 26 gauge stainless steel internal cannula (PlasticsOne) projecting 0.5 $\mathrm{mm}$ beyond the tip of the guide cannula was connected to a syringe pump (Harvard Apparatus). The AMPA receptor antagonist and the NMDA receptor antagonist were infused into the vHPC in a volume of $0.5 \mu \mathrm{l}$ at a rate of $0.1 \mu \mathrm{l}$ per minute. The internal cannula was withdrawn $5 \mathrm{~min}$ after the end of infusion, and animals underwent social testing $30 \mathrm{~min}$ after drug infusion. Testing took place over $4 \mathrm{~d}$, and each day an individual mouse received only one drug, counterbalanced for treatment and stimulation (laser ON or OFF) order. Glutamate receptor antagonism consisted of a mixture of $22 \mathrm{~mm}$ of NBQX and $38 \mathrm{~mm}$ of AP5 (Tocris Bioscience), dissolved in $0.9 \%$ saline, and prepared freshly on each day of the experiment.

\section{Immunohistochemistry}

To use c-fos expression as a readout for neural activity, mice expressing ChR2(H134R)-eYFP or eYFP were stimulated in vivo inside their home cage for $3 \mathrm{~min}$ (with the same stimulation protocol used for behavioral testing) $90 \mathrm{~min}$ before death. All mice were anesthetized with sodium pentobarbital and transcardially perfused with ice-cold $4 \%$ PFA in $1 \times$ PBS, pH 7.3. The brain was extracted and postfixed in 4\% PFA overnight, followed by transfer to $30 \%$ sucrose in $1 \times$ PBS. Brains were sectioned into $40-\mu \mathrm{m}$-thick coronal sections using a sliding microtome (HM430; Thermo Fisher Scientific) and stored in PBS at $4_{\text {[GRAPHIC] }}$ before being processed using immunohistochemistry. Sections were blocked in Triton $\mathrm{X}-1000.3 \% / \mathrm{PBS}$ and 3\% normal donkey serum for $1 \mathrm{~h}$ at room temperature, followed by incubation with primary antibody (rabbit anti-c-fos 1:500; Calbiochem) for $17-20 \mathrm{~h}$ at $4^{\circ} \mathrm{C}$. Sections were then washed 4 times for 10 min each with PBS before and after incubation with secondary antibody (AlexaFluor-647 anti-rabbit, 1:500, Invitrogen) for $2 \mathrm{~h}$ at room temperature. Sections were then incubated with a DNA specific fluorescent probe (DAPI; 1:50,000) for $30 \mathrm{~min}$ and washed 4 times with $1 \times$ PBS followed by mounting on microscope slides with PVDDABCO (Sigma).

\section{Confocal microscopy}

Confocal fluorescence images were acquired using an Olympus FV1000 confocal laser scanning microscope using a $10 \times / 0.40 \mathrm{NA}$ or a $40 \times / 1.30$ NA oil-immersion objective. Serial $z$-stack images covering a depth of 10 $\mu \mathrm{m}$ through multiple sections were acquired using the image analysis software (Fluoview, Olympus). The number of c-fos-positive cells were counted by experimenters blind to the experimental conditions. Mice showing eYFP somata expression in the cortex were excluded from analysis.

\section{Statistics}

A two-way ANOVA was used to examine group differences followed by Bonferroni post hoc tests. We performed a Bonferroni correction for multiple comparisons by multiplying the $p$ value for each individual test by the number of comparisons made. For all results, significance threshold was placed at $p=0.05$ and $p<0.01$. All data are shown as \pm SEM.

\section{Results}

\section{Optogenetic inhibition of BLA inputs to the vHPC increases social behaviors}

Using $\mathrm{AAV}_{5}$ under the control of the CaMKII $\alpha$ promoter, we transduced BLA pyramidal neurons with an enhanced version of halorhodpsin (eNpHR3.0) (Gradinaru et al., 2010). In experimental groups, BLA projection neurons were transduced with $\mathrm{NpHR}$ fused to an eYFP (AAV ${ }_{5}$-CaMKII $\alpha$-NpHR-eYFP), whereas control animals received the same viral vector carrying the fluorophore alone $\left(\mathrm{AAV}_{5}-\mathrm{CaMKII} \alpha\right.$-eYFP). To inhibit NpHRexpressing BLA axon terminals in the vHPC, we bilaterally implanted optical fibers above the vHPC to allow for the delivery of 594 nm light to the pyramidal layer of the vHPC (Fig. 1A). The mice were then tested on a well-validated social interaction test, the residentintruder procedure (File and Pope, 1974; Winslow, 2003; Silverman

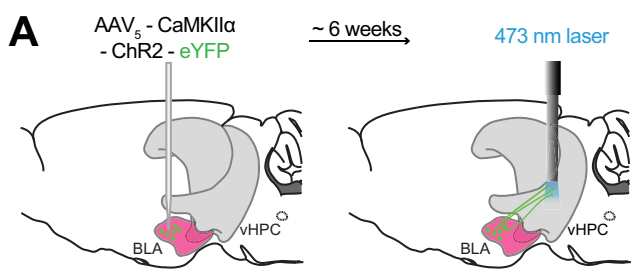

B
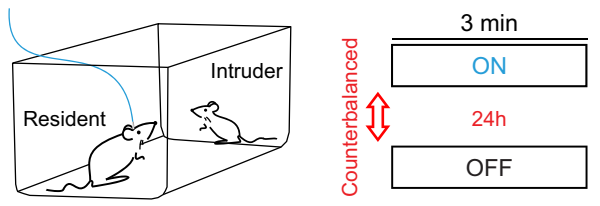

C ChR2:BLA-vHPC $(\mathrm{n}=8)$ eYFP:BLA-vHPC $(\mathrm{n}=8)$
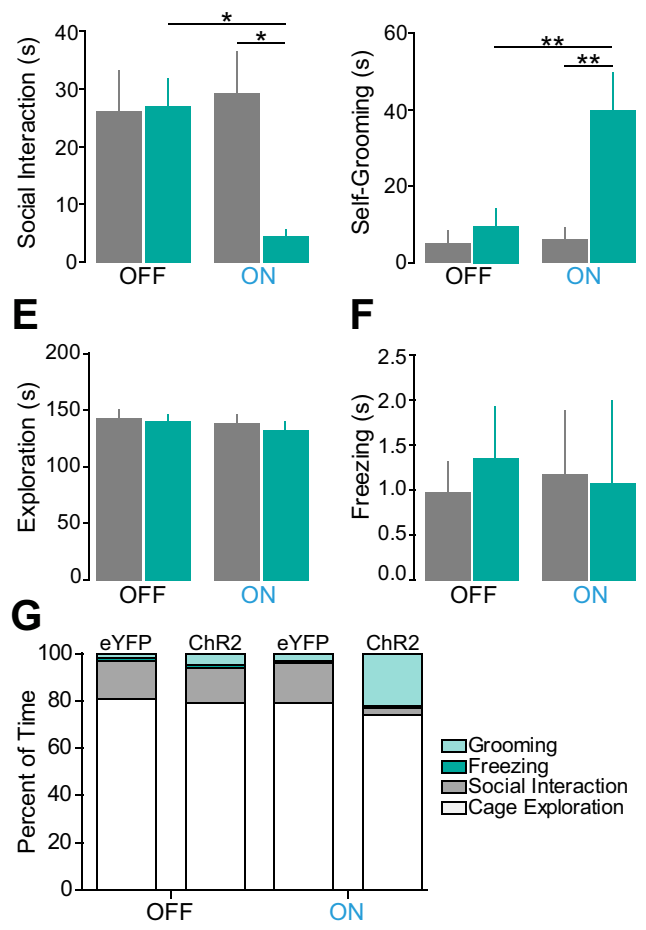

Figure 3. Activation of BLA axon terminals in the $\mathrm{VHPC}$ with ChR2 decreases social interaction in a resident-juvenile intruder procedure. Glutamatergic neurons from the BLA were transduced with either ChR2-eYFP $(n=8)$ or eYFP control $(n=8)$. After $\sim 6-7$ weeks of incubation after viral transduction of BLA cell somata, blue light was delivered ( $3 \mathrm{~min}, 20 \mathrm{~Hz}, 5 \mathrm{~ms}$ pulses) via a unilateral optical fiber implanted above the VHPC. $A$, Left, Sagittal view brain schematic indicating viral injection into the BLA. Right, Schematic indicating unilateral optical fiber location into the VHPC. Top, Experimental timeline. $\boldsymbol{B}$, Schematic indicating the home-cage resident-juvenile (3-4 weeks) intruder behavioral procedure. Three minute epochs were counterbalanced for order with a $24 \mathrm{~h}$ interval between $0 \mathrm{~N}$ and $\mathrm{OFF}$ light conditions. Novel juvenile intruders were used for each epoch. C, ChR2 mice spent significantly less time (seconds) performing social interaction than eYFP mice during the blue light illumination epoch. ${ }^{*} p=0.034$. $\boldsymbol{D}$, ChR2 mice also spent significantly more time (seconds) performing self-grooming than eYFP mice in the presence of a juvenile intruder. ${ }^{* *} p=0.008$. $\boldsymbol{E}$, No significant effect of light stimulation or group was detected for the time spent exploring their home cage. $\boldsymbol{F}$, No significant effect of light stimulation or group was detected in freezing behavior in the presence of a juvenile intruder. G, Percentage of total time (3 $\mathrm{min})$, showing social interaction, selfgrooming, cage exploration, and freezing. Data are mean values; error bars indicate \pm SEM.

et al., 2010; Himmler et al., 2013). Each mouse was tested on two separate days, with different juvenile intruders (3-4 weeks old), to allow for within-subject and within-session comparisons as well as group comparisons Each mouse had $1 \mathrm{~min}$ of habituation in their home cage followed by a 3 min epoch of either light-off (OFF) or 
A
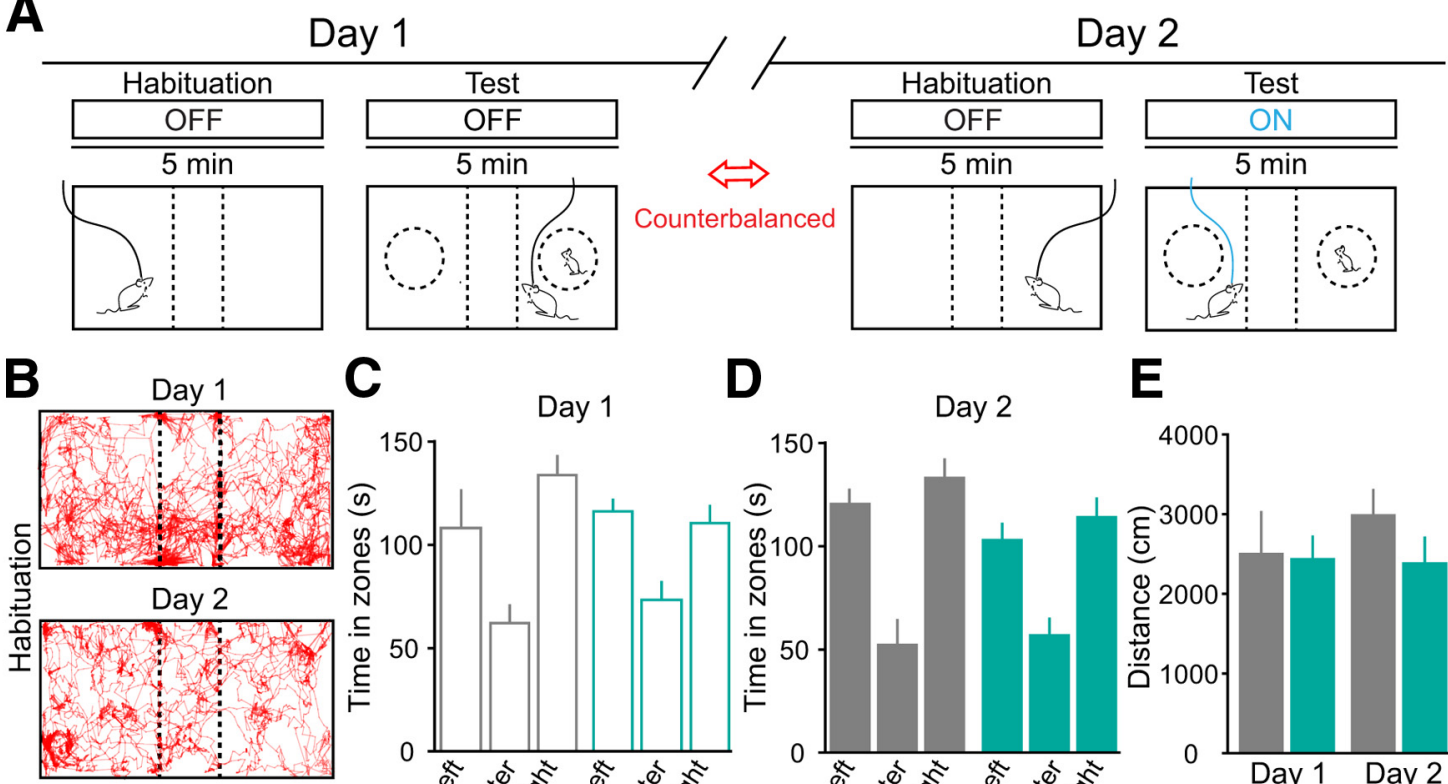

D

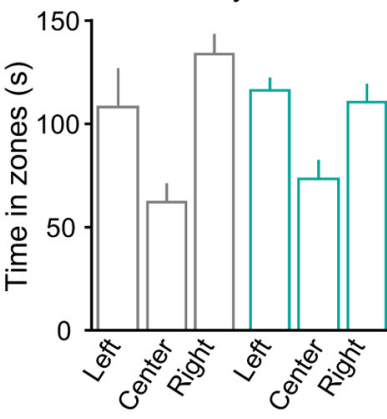

Day 2

$\mathbf{E}$
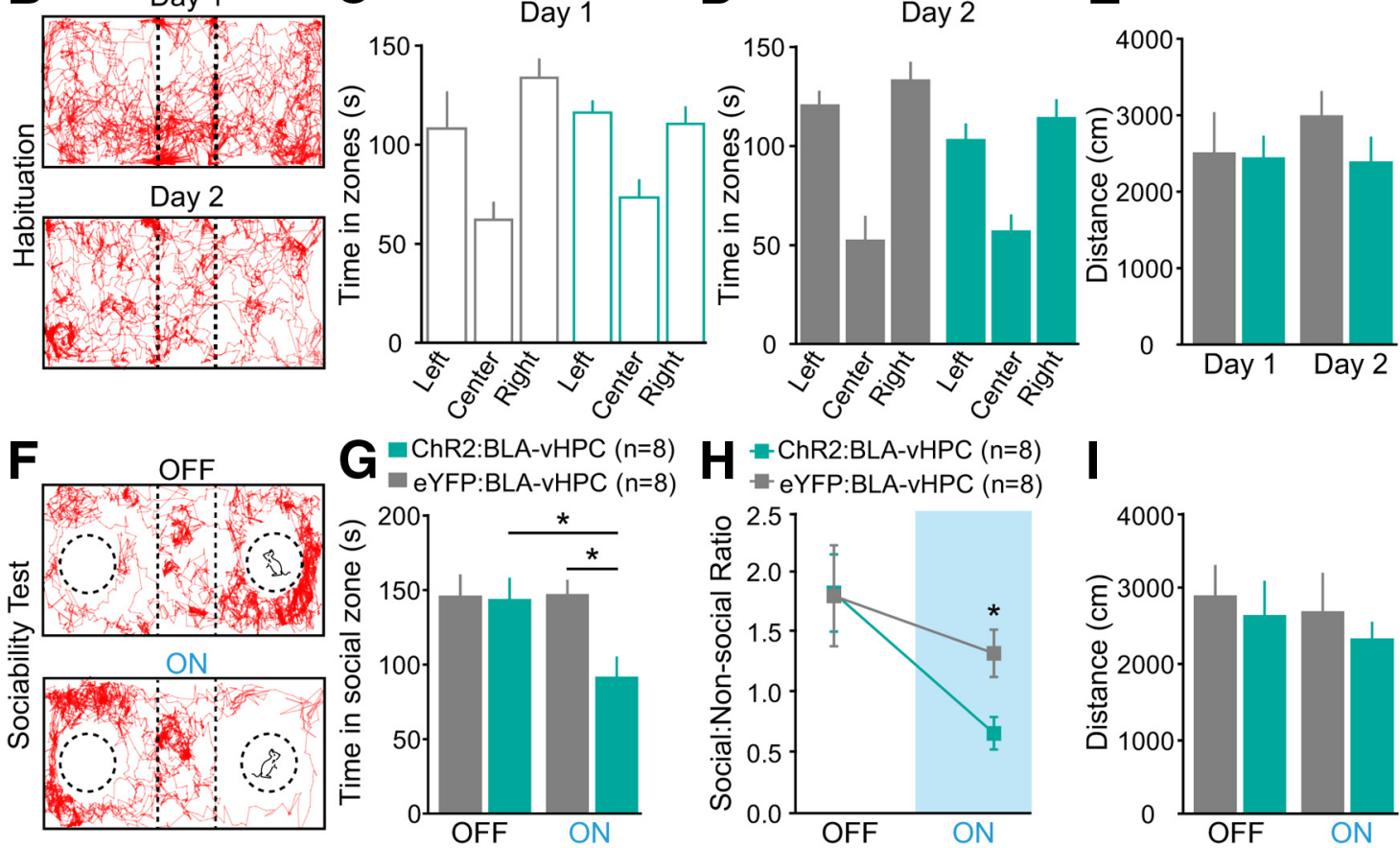

Figure 4. Activation of BLA projections to the VHPC with ChR2 decreases sociability in a three chamber sociability test. In a different group of animals from Figure 3 , glutamatergic neurons from the BLA were transduced with either ChR2-eYFP $(n=8)$ or eYFP control $(n=8)$. After $\sim 6-7$ weeks of viral transduction of BLA cell somata, blue light was delivered $(5 \mathrm{~min}, 20 \mathrm{~Hz}, 5 \mathrm{~ms}$ pulses) via unilateral optical fiber implanted in the VHPC. A, Top, Timeline across $2 \mathrm{~d}$ of testing. Bottom, Schematic indicating the three chamber sociability test and 5 min experimental epochs. Each testing day was composed of habituation to the arena followed by $5 \mathrm{~min}$ of laser ON or laser OFF stimulation (counterbalanced for order). Different juvenile mice were used for each testing epoch, counterbalanced for side of the testing arena. $\boldsymbol{B}$, Representative animal tracks during the habituation phase for day 1 (top) and day 2 (bottom). $\boldsymbol{C}$, Time spent in the different zones of the arena during day 1. No significant differences were found between the times spent on the right chamber versus the left chamber. $\boldsymbol{D}$, Time spent in the different zones of the arena during day 2 . No significant differences were found between the times spent on the right chamber versus the left chamber. $\boldsymbol{E}$, No significant effect on locomotion during the habituation phase during the $2 \mathrm{~d}$ of testing. $\boldsymbol{F}$, Representative animal tracks during the testing sessions for OFF (top) and ON (bottom). G, ChR2 mice spent significantly less time (seconds) in the social zone relative to eYFP mice during the blue light illumination epoch. ${ }^{*} p=0.033 . \boldsymbol{H}$, ChR2 also showed a significantly lower social/nonsocial ratio during laser stimulation compared with eYFP mice. ${ }^{*} p=0.023 . I$, No significant effect of locomotion during the habituation phase during the laser stimulation. Data are mean values; error bars indicate \pm SEM.

light-on (ON) illumination using constant illumination with 594 nm light. During the testing epochs, the residents were presented with a juvenile intruder. Groups were counterbalanced for order of light stimulation (Fig. 1B).

Mice in the NpHR group showed significantly greater total investigation of the juvenile intruder, reflecting an increase in social behaviors, relative to eYFP mice during the ON epoch (Fig. $1 C$; two-way ANOVA demonstrating group $\times$ light epoch interaction, $F_{(1,13)}=12.89, p=0.0033$; Bonferroni post hoc analysis, corrected for multiple comparisons, $p=0.046)$. No significant differences were found in stereotypical behaviors, such as selfgrooming (Fig. 1D; two-way ANOVA did not detect a group $\times$ light epoch interaction, $\left.F_{(1,13)}=0.02, p=0.8993\right)$. Mice in the NpHR group also displayed a decrease in time spent performing home-cage exploration during the ON epoch (Fig. 1E; two-way ANOVA demonstrating group $\times$ light epoch interaction, $F_{(1,13)}=$ $7.12, p=0.0193$; Bonferroni post hoc analysis, corrected for mul- tiple comparisons, $p=0.035$ ), perhaps explained by the increased attention to the juvenile intruder. No significant differences were found in freezing (immobilization) behavior (Fig. 1F; two-way ANOVA did not detect a group $\times$ light epoch interaction, $\left.F_{(1,13)}=0.52, p=0.4839\right)$. Each epoch session lasted $3 \mathrm{~min}$. Figure $1 G$ shows the overall time mice spent engaging in different behaviors for experimental and control groups. NpHR mice spent significantly more time exploring the intruder than eYFP controls. These data demonstrate that silencing BLA inputs to the vHPC increased the time spent on social interaction. Next, we explored whether the ability of BLA inputs to the vHPC could control social interaction in a bidirectional manner. After completion of the $2 \mathrm{~d}$ of behavior, brains were extracted and the tissue was prepared for confocal microscopy. In Figure $2 A$, we show the locations of viral injections (Fig. $2 A$ ) and representative confocal images from an NpHR mouse (Fig. 2B). Optic fiber placements were also verified for all animals (Fig. 2C). Representative confo- 

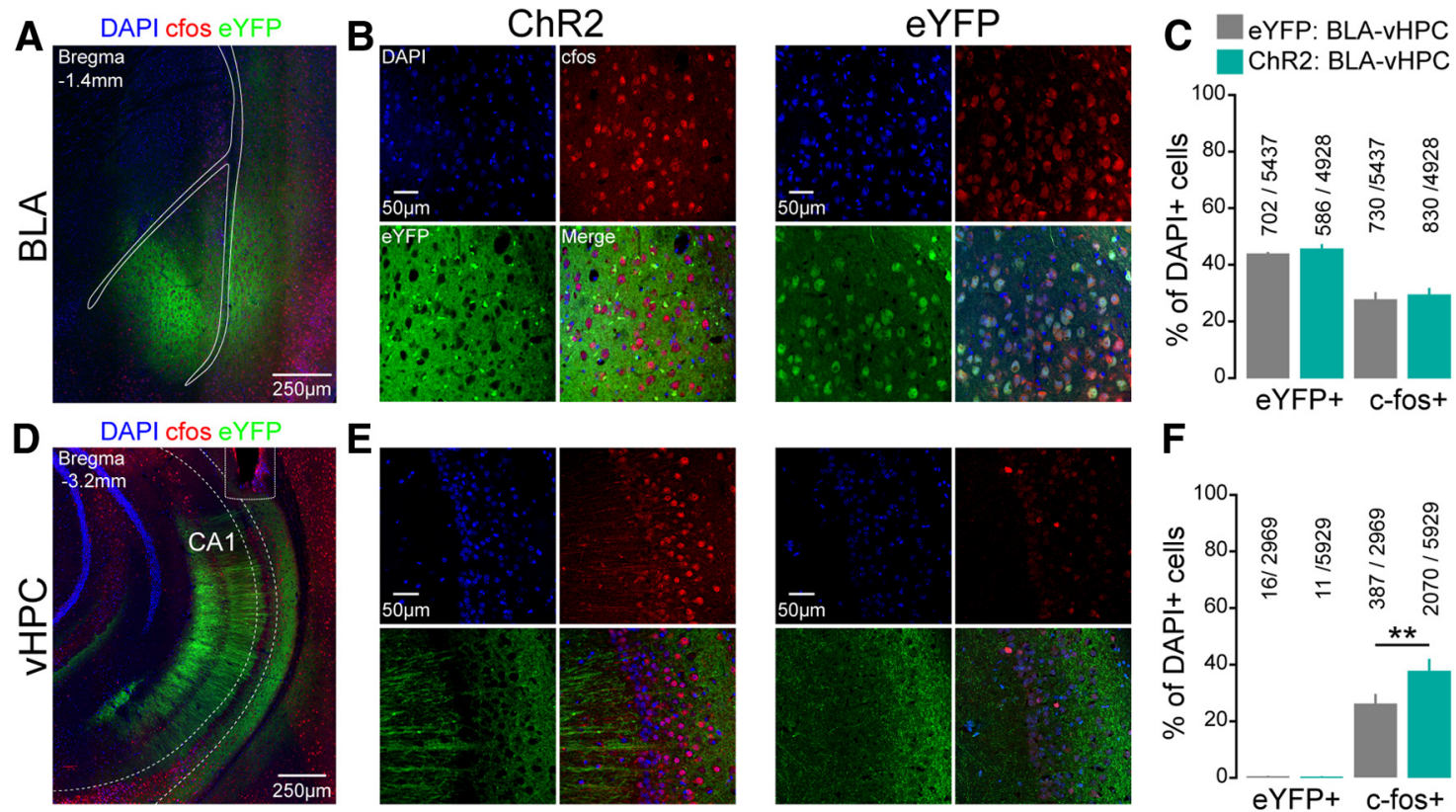

Figure 5. Activation of BLA axon terminals in the vHPC using ChR2 increases $c$-fos expression in the pyramidal layer of vHPC. Blue represents DAPI; green, eYFP; red, $c$-fos. $A$, Confocal image of the BLA of representative ChR2 animal. $B$, Confocal images of the BLA from two representative mice. Representative ChR2:BLA-vHPC animal (left) and representative eYFP:BLA-vHPC mice (right). C, Percentage of DAPI-positive $(+)$ cells expressing eYFP or $c$-fos in the BLA $\left(n=8 \mathrm{ChR2}\right.$ mice and $n=8 \mathrm{eYFP}$ mice). No differences between groups were found in c-fos ${ }^{+}$or eYFP ${ }^{+}$cells in the BLA. D, Confocal image of the VHPC from representative ChR2 mice. $\boldsymbol{E}$, vHPC confocal images of two representative mice. Representative ChR2:BLA-vHPC animal (left) and representative eYFP:BLA-vHPC mice (right). $\boldsymbol{F}$, Percentage of DAPI-positive $(+)$ cells expressing eYFP or c-fos in the vHPC ( $n=8$ ChR2 mice and $n=8 \mathrm{eYFP}$ mice). Compared with eYFP:BLA-vHPC controls, light stimulation of BLA terminals in the vHPC increased the percentage of $c-f o s(+)$ cells in the vHPC of ChR2:BLA-vHPC group. Data are mean \pm SEM. ${ }^{* *} p=0.0028$.

cal images from an NpHR mouse's optic fiber position are presented in Figure 2D.

\section{Optogenetic activation of BLA inputs to the vHPC decreases social behaviors}

We transduced BLA pyramidal neurons with a channelrhodopsin-2 (ChR2)-eYFP fusion protein in experimental animals and eYFP alone in control animals along with a unilaterally implanted optical fiber over the vHPC on the ipsilateral hemisphere (Fig. $3 A$ ) before testing in the resident-intruder procedure (Fig. $3 B$ ). We used the same procedure as in the previous experiments, but with the illumination epoch consisting of a continuous train at $20 \mathrm{~Hz}$ of $473 \mathrm{~nm}$ (blue) light pulses, with $5 \mathrm{~ms}$ pulse duration. ChR2 mice showed reduced investigation of the juvenile intruder, reflecting a reduction in social interaction (Fig. 3C; two-way ANOVA demonstrating group $\times$ light epoch interaction, $F_{(1,14)}=9.04, p=0.0094$; Bonferroni post hoc analysis, corrected for multiple comparisons $p=0.034)$. ChR2 mice also showed a significant increase in self-grooming compared with eYFP controls (Fig. 3D; two-way ANOVA demonstrating group $\times$ light epoch interaction, $F_{(1,14)}=14.90, p=0.0017$; Bonferroni post hoc analysis, corrected for multiple comparisons, $p=0.008$ ). No differences were found in home-cage exploration (Fig. 3E; twoway ANOVA did not detect a group $\times$ light epoch interaction, $F_{(1,14)}=0.04, p=0.8497$ ) and freezing behavior (Fig. 3F; twoway ANOVA did not detect a group $\times$ light epoch interaction, $\left.F_{(1,14)}=0.29, p=0.5975\right)$. Figure $4 G$ shows the overall time ChR2 and eYFP groups spent engaging in different behaviors during the test. As shown above, ChR2 mice spent less time exploring the intruder compared with eYFP controls.

To evaluate other aspects of social behavior, we also tested a different group of mice on the three chamber sociability test (Fig. 4). To allow for within-subject comparisons in addition to between-group comparisons, we tested each mouse on two sep- arate days on the three chamber sociability test with different juvenile intruders (3-4 weeks) each day (Fig. 4A). Each test had 5 min of habituation in the testing arena. Representative ChR2 mouse tracks are shown in Figure 3B. No differences were found in the time spent in the right zone versus the left zone of the testing chamber (Fig. 4C; two-way ANOVA did not detect a group $\times$ light epoch interaction, $F_{(1,14)}=1.69, p=0.2146$; and Fig. $4 D$; two-way ANOVA did not detect a group $\times$ light epoch interaction, $\left.F_{(1,14)}=0.09, p=0.7646\right)$. No differences were found in distance traveled (Fig. 4E; two-way ANOVA did not detect group $\times$ light epoch interaction, $F_{(1,14)}=0.93, p=$ 0.3503 ). The habituation period was followed by one $5 \mathrm{~min}$ epoch of either a baseline epoch without illumination (OFF) or illumination using continuous train at $20 \mathrm{~Hz}$ of $473 \mathrm{~nm}$ (blue) light pulses, with $5 \mathrm{~ms}$ pulse duration $(\mathrm{ON})$. During the testing epochs, the test mouse was presented with a juvenile intruder inside an inverted cup for which a representative animal track from the ChR2 group is shown (Fig. 4F). Groups were counterbalanced for order of light stimulation. Mice in the ChR2 group displayed a decrease in time spent in the social zone upon illumination $(\mathrm{ON}$ epoch) relative to eYFP controls (Fig. 4G; two-way ANOVA demonstrating group $\times$ light epoch interaction, $F_{(1,14)}=9.04, p=$ 0.0094; Bonferroni post hoc analysis, corrected for multiple comparisons, $p=0.033$ ). ChR2 mice also showed a lower social/ nonsocial ratio during the $\mathrm{ON}$ epoch, which indicates that during light stimulation ChR2 mice spent more time investigating the nonsocial zone (Fig. 4H; two-way ANOVA demonstrating group $\times$ light epoch interaction, $F_{(1,14)}=8.02, p=0.0133$; Bonferroni post hoc analysis, corrected for multiple comparisons, $p=$ 0.023). Furthermore, we simultaneously analyzed locomotor activity across epochs and did not detect any changes during the $\mathrm{ON}$ epoch relative to the OFF epoch as measured by distance traveled (Fig. 4I; two-way ANOVA did not detect a group $\times$ light epoch interaction, $\left.F_{(1,14)}=0.03, p=0.8728\right)$. 


\section{Optogenetic stimulation of BLA axon terminals in the vHPC increases c-fos expression in the vHPC, but not in BLA somata}

To test for the effects of blue light illumination on neuronal activation, we used the immediate early gene $\mathrm{c}$-fos as a readout for neural activity. A confocal image of the BLA from a representative ChR2 mouse is shown in Figure 5A, demonstrating basal c-fos expression (red) within the BLA. Confocal images of the pyramidal layer in vHPC CA1 from representative ChR2 and eYFP mice are shown in Figure $5 B$. We did not observe a change in BLA somata $\mathrm{c}$-fos expression induced by illumination of BLA terminals in the vHPC relative to eYFP control mice (Fig. $5 C$; unpaired Student's $t$ test, $\mathrm{df}=14, t=0.4515, p=0.3293$ ). Although light stimulation does not alter basal activity on the BLA, we also show that c-fos expression was increased in the pyramidal layer of the vHPC CA1 extending to $\sim 1.5 \mathrm{~mm}$ below the fiber tip (Fig. $5 D, E)$. Quantification of c-fos demonstrates a significant increase of cell activity on the vHPC during light stimulation (Fig. $5 F$; unpaired Student's $t$ test, $\mathrm{df}=17, t=3.166, p=0.0028$ ). Confocal images were also taken from each animal to confirm the viral injection site (Fig. 6A) and optic fiber position (Fig. 6B).

\section{BLA excitatory projections to the vHPC are sufficient to mediate changes in social behaviors}

Although our data suggest that excitation of glutamatergic inputs from the BLA to the vHPC can reduce social behaviors, the illumination of ChR2-expressing terminals in the vHPC could induce depolarization of axons of passage and/or back-propagating action potentials to BLA somata (Petreanu et al., 2007). To control for this possibility, we combined in vivo pharmacological manipulations with our in vivo optogenetic manipulations during the resident-intruder procedure (Fig. 7). Once again, we expressed ChR2 in BLA neurons but unilaterally implanted a guide cannula rather than an optic fiber to deliver either saline or glutamate receptor antagonists to the $\mathrm{VHPC} \sim 30 \mathrm{~min}$ before testing and laser stimulation on the resident-intruder procedure (Fig. $7 A$ ). To allow for a within-subject comparison, we tested each animal four times on different days, with unilateral administration of either saline or a combination of the AMPA receptor antagonist NBQX (22 mM) and NMDA receptor antagonist AP5 (38 mM), counterbalanced for order. This enabled comparison of saline trials to glutamate receptor antagonist mixture (GluRX) trials (Fig. 7B). Different intruders were used for each day of testing.

In saline trials, mice replicated the light-induced reduction of social interaction on the resident-intruder procedure; but after treatment with glutamate receptor antagonists, the light-induced changes in social interaction were attenuated (Fig. 7C; two-way ANOVA demonstrating group $\times$ light epoch interaction, $F_{(1,14)}=$ 10.37, $p=0.0062$; Bonferroni post hoc analysis, corrected for multiple comparisons, $p=0.048$ ). Furthermore, the lightinduced increase in grooming evident in the saline group was also attenuated by the GluRX treatment (Fig. 7D; two-way ANOVA demonstrating group $\times$ light epoch interaction, $F_{(1,14)}=9.62$, $p=0.0078$; Bonferroni post hoc analysis, corrected for multiple comparisons, $p=0.036$ ). No differences were found between groups in locomotor activity shown by distance traveled (Fig. 7E; two-way ANOVA did not detect a group $\times$ light epoch interaction, $F_{(1,14)}=0.56, p=0.4674$ ) or freezing behavior (Fig. $7 F$; two-way ANOVA did not detect a group $\times$ light epoch interaction, $\left.F_{(1,14)}=1.97, p=0.1827\right)$. Figure $7 G$ shows the overall time ChR2:GluRX and ChR2:saline groups spent engaging in different behaviors during the test. As shown above, the light-induced
A Viral injection in BLA:

B Optic fiber tip in vHPC:

-AAV ${ }_{5}$-CaMKIIa-ChR2-eYFP $(n=16)$

-AAV ${ }_{5}$-CaMKIla-eYFP $(\mathrm{n}=16)$

$\times$ ChR2: BLA-vHPC $(n=16)$

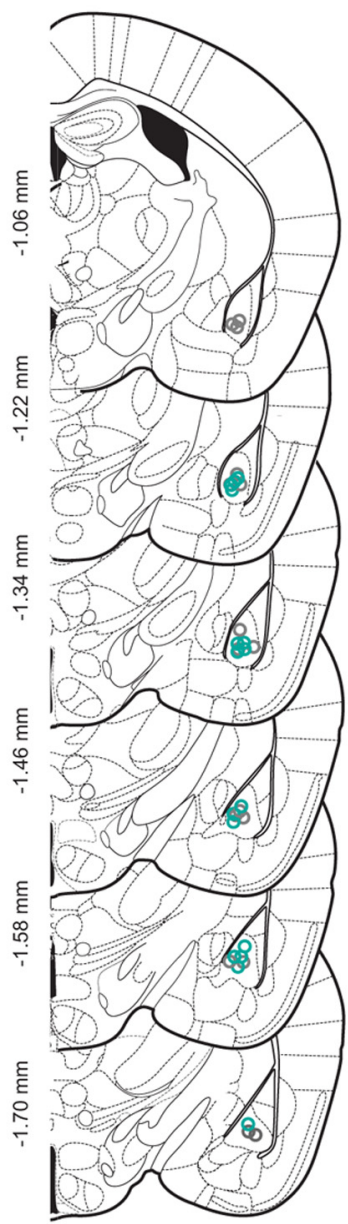

$\times$ eYFP:BLA-vHPC $(n=16)$

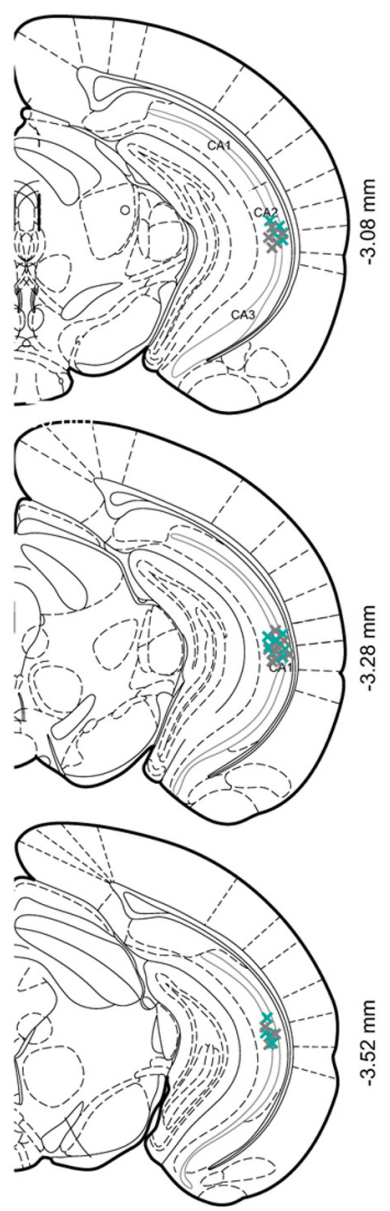

Figure 6. Histologically verified placements of viral injections and optical fiber tips in ChR2: BLA-vHPC and EYFP:BLA-vHPC groups. $\boldsymbol{A}$, Coronal sections from bregma of the BLA. Center of the viral injections in the BLA for all the mice injected with ChR2 ( $n=16$; green circles) and eYFP ( $n=16$; gray circles). $\boldsymbol{B}$, Coronal sections from bregma of the VHPC. Location of the optic fibers tip above the pyramidal layer of vHPC for ChR2:BLA-vHPC (green crosses) and eYFP (gray crosses).

changes were replicated in the ChR2:saline group and attenuated with the presence of the GluRX. After behavioral testing, animals were processed to allow for histological verification of injection site (Fig. 8A) and cannula placement (Fig. 8B).

\section{Discussion}

Our results demonstrate a critical role for BLA projections to the vHPC in bidirectionally modulating social interaction. Additionally, activation of this pathway increases self-grooming while decreasing social interaction. These findings provide broad implications for the involvement of this pathway in behaviors relevant to autism spectrum disorders, obsessive-compulsive disorder, and social anxiety, and represent a potential new target for therapeutic development.

Strong evidence suggests a functional role for the amygdala in social processing or social cognition in humans (Killgore and Yurgelun-Todd, 2005; Schultz, 2005; Bickart et al., 2011), as well as in social behavior in animals (Ferguson et al., 2001; Amaral, 2002). However, support for a role of the vHPC has been less 
A

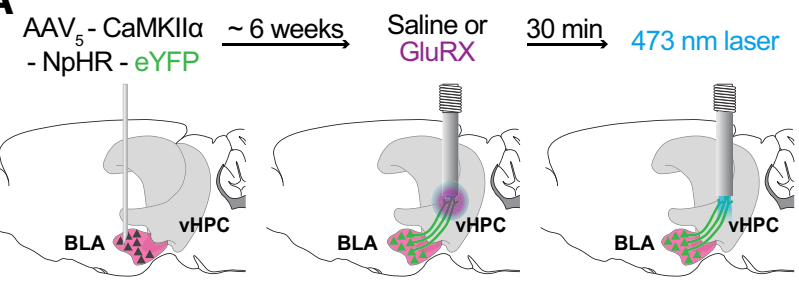

B
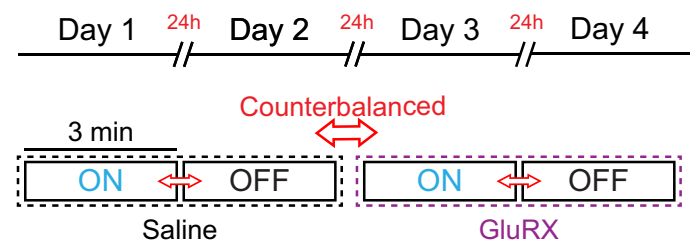

C $\square$ GluRX:ChR2 $(n=8)$
Saline:ChR2 $(n=8)$

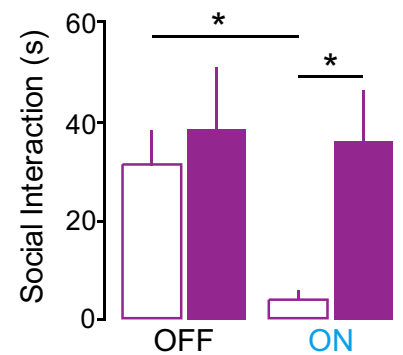

E

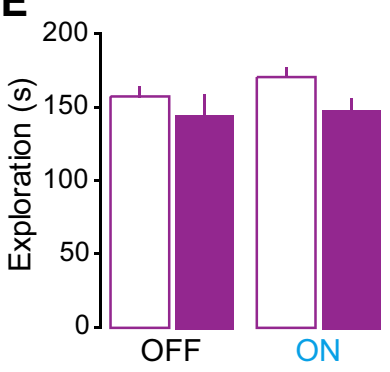

\section{D}

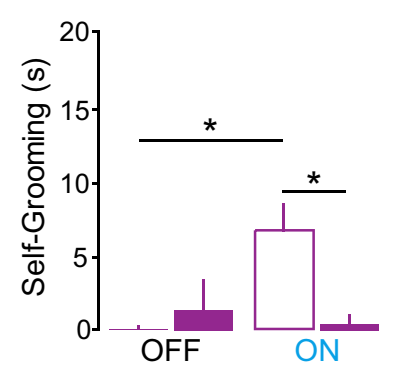

$\mathbf{F}$

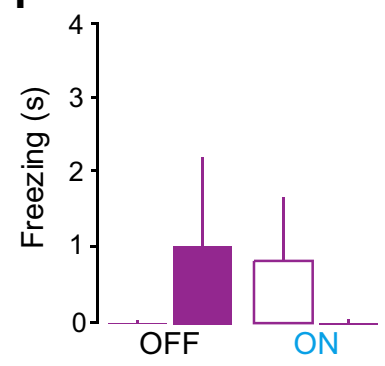

G

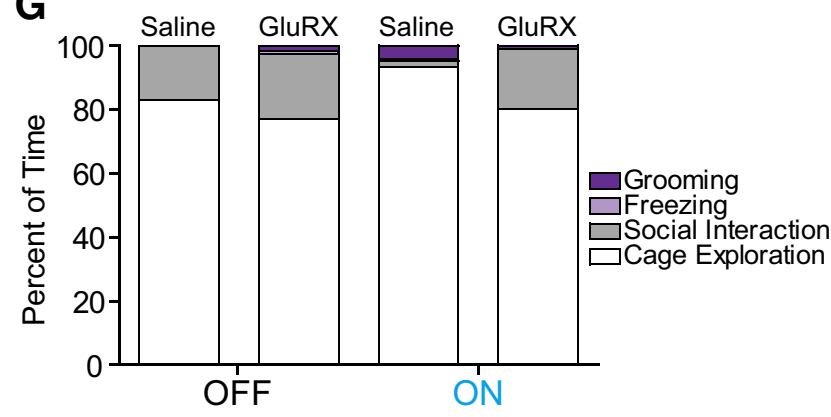

Figure 7. Activation of BLA inputs to the VHPC is sufficient to mediate changes in social interaction without affecting the ability to explore the environment. $A$, Glutamate receptor antagonists (GluRX:AP5 +NBQX, purple) or saline (black) were unilaterally infused into the vHPC using the same guide cannula subsequently used for light delivery via an acutely inserted optical fiber. Top, Experimental timeline. Left, Sagittal brain schematic indicating viral injections into the BLA. Middle, Sagittal schematic indicating unilateral cannula location into the vHPC. Right, Sagittal schematic of removable optical fiber used for light delivery $30 \mathrm{~min}$ after GluRX delivery. B, Top, Testing took place over 4 consecutive days. Schematic of 3 min epochs where mice received either drug or saline treatment. All experiments where counterbalanced for treatment and stimulation ( $\mathrm{ON}$ or $\mathrm{OFF}$ epoch) order. Novel juvenile intruders were used for each session. C, GluRX attenuated the light stimulation effect. GluRX:ChR2 mice $(n=8)$ spent significantly more time (seconds) performing social interaction than Saline:ChR2 mice $(n=8)$

A $\circ \mathrm{AAV}_{5}$-CaMKIla-ChR2-eYFP $(n=8)$

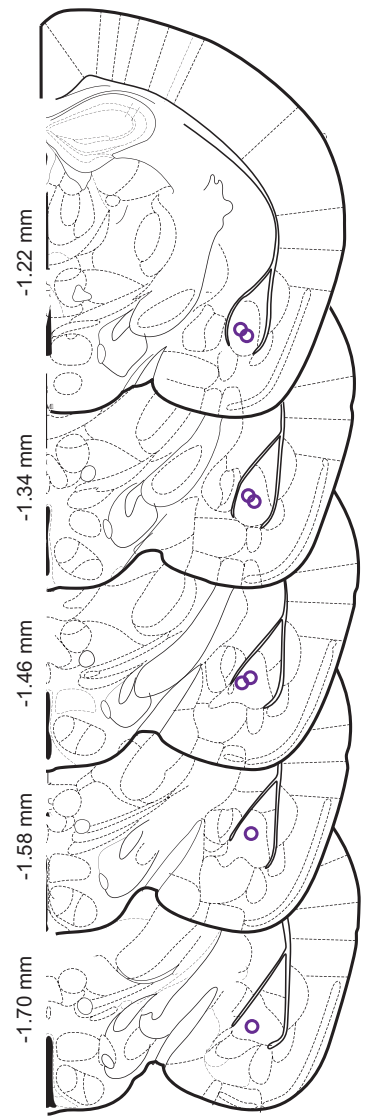

B $\times$ guide-cannula tip $(n=8)$

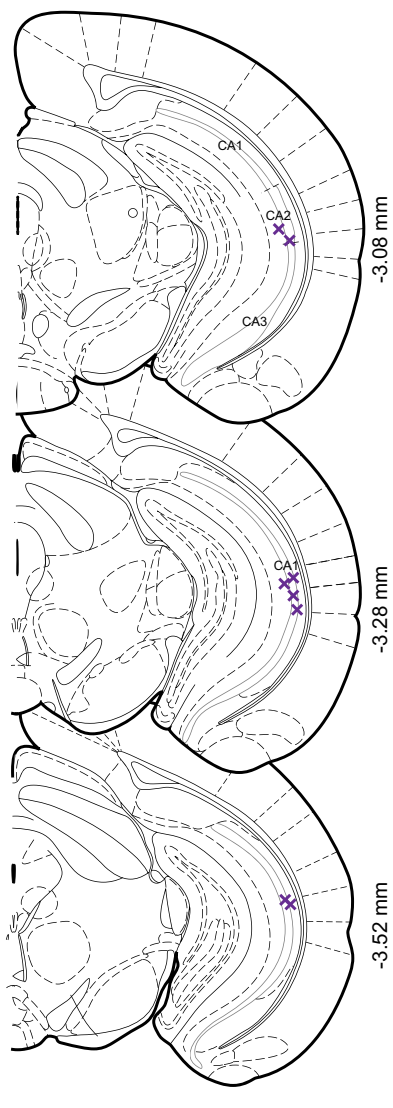

Figure 8. Histologically verified placements of viral injections and optical fiber tips in ChR2 animals used for pharmacology experiments. $\boldsymbol{A}$, Coronal sections of the BLA with coordinates reflecting anteroposterior distance from bregma. Center of viral injections in the BLA for all mice expressing ChR2 ( $n=8$; purple circles). B, Coronal sections of the vHPC with coordinates from bregma. Location of cannulae above the pyramidal layer of vHPC for ChR2 animals (purple crosses).

clear. It has been reported that increases in 5HT and cAMP are observed in the vHPC after social interaction (Cadogan et al., 1994). However, their study used a novel arena, which may have been anxiogenic, which is in contrast to the home-cage social interaction test but similar to the three chamber sociability test. Although these changes appear distinct from our observation of increased c-fos expression, our findings show that the same photostimulation that reduced social interaction showed increased vHPC activity and agree with this report in terms of linking social interaction to changing neural dynamics within the vHPC. In another study, oxytocin was recently shown to increase the signal-to-noise ratio of CA1 pyramidal neurons in mice in an $e x$ vivo preparation (Owen et al., 2013), providing one possible mechanism underlying the ability of oxytocin to reduce social deficits associated with autism spectrum disorders (Guastella et

$\leftarrow$

during the blue light illumination epoch. ${ }^{*} p=0.048$. D, GluRX:ChR2 mice also spent significantly less time performing self-grooming compared with Saline:ChR2 mice. ${ }^{*} p=0.036$. $E$, No significant effect of light stimulation or group was detected on the time spent exploring their home cage. $\boldsymbol{F}$, No significant effect of light stimulation or group was detected for freezing behavior in the presence of a juvenile intruder. $\mathbf{G}$, Percentage of total time (3 min), showing social interaction, self-grooming, cage exploration, and freezing. Data are mean \pm SEM. 
al., 2010) and to ameliorate the symptoms of obsessive-compulsive disorder (Ansseau et al., 1987) in humans.

Despite these findings, there has been contention about whether the vHPC has a critical role in social interaction. Specifically, although ibotenic acid lesions in neonatal rats produced dramatic changes in social interaction when the rats were tested in adulthood in the absence of changes in general anxiety-related behaviors, no such changes in social interaction were observed when the lesions were performed in adult rats (SamsDodd et al., 1997; Becker et al., 1999). This result suggests that the vHPC does not play an active role in social interaction in adulthood. However, these lesions were nonspecific and may have targeted multiple circuits in the vHPC with opposing functions in social interaction, thereby producing a zero sum effect on behavior. Furthermore, in these studies, the lesions were performed 2 weeks before testing, and compensatory mechanisms in the adult brain may have contributed to the lack of change in social interaction observed. In contrast to this nonspecific ablation of the vHPC region, in this study we transiently modulated the activity across a specific subpopulation of synapses in the vHPC without causing any permanent damage. Whereas in vivo electrophysiological studies have been performed in the BLA (Wang et al., 2011) and vHPC during anxiety (Adhikari et al., 2010), and recordings have also been performed in the BLA during social behaviors (Katayama et al., 2009), our findings provide the first evidence that BLA inputs to the vHPC have a causal relationship with social behavior. Furthermore, the neural encoding dynamics of vHPC-projecting BLA neurons have yet to be revealed during social interaction.

In our previous work, we identified two projections originating from the BLA that showed opposing effects on anxiety-related behavior (Tye et al., 2011; Felix-Ortiz et al., 2013). Here, we extend these findings by showing that BLA projections to the vHPC not only mediate anxiety-related behaviors but also mediate social behaviors. We speculate that the dual function of this pathway may contribute to the high rate of comorbidity of autism and anxiety disorders (Kim et al., 2000; Amaral and Corbett, 2008), as individuals with both autism and anxiety-related symptoms could have a perturbation in this pathway.

\section{References}

Adhikari A, Topiwala MA, Gordon JA (2010) Synchronized activity between the ventral hippocampus and the medial prefrontal cortex during anxiety. Neuron 65:257-269. CrossRef Medline

Amaral DG, Corbett BA (2008) The amygdala, autism and anxiety. In: Autism: neural basis and treatment possibilities (Bock Organizer G, Goode J, eds), pp 177-197. New York: Wiley.

Amaral DG (2002) The primate amygdala and the neurobiology of social behavior: implications for understanding social anxiety. Biol Psychiatry 51:11-17. CrossRef Medline

American Psychiatric Association (2013) Diagnostic and statistical manual of mental disorders, Ed 5. Arlington, VA: American Psychiatric Association.

Ansseau M, Legros JJ, Mormont C, Cerfontaine JL, Papart P, Geenen V, Adam F, Franck G (1987) Intranasal oxytocin in obsessive-compulsive disorder. Psychoneuroendocrinology 12:231-236. CrossRef Medline

Becker A, Grecksch G, Bernstein HG, Höllt V, Bogerts B (1999) Social behaviour in rats lesioned with ibotenic acid in the hippocampus: quantitative and qualitative analysis. Psychopharmacology 144:333-338. CrossRef Medline

Bickart KC, Wright CI, Dautoff RJ, Dickerson BC, Barrett LF (2011) Amygdala volume and social network size in humans. Nat Neurosci 14: 163-164. CrossRef Medline

Bunnell BN, Sodetz FJ Jr, Shalloway DI (1970) Amygdaloid lesions and social behavior in the golden hamster. Physiol Behav 5:153-161. CrossRef Medline
Cadogan AK, Kendall DA, Fink H, Marsden CA (1994) Social interaction increases 5-HT release and cAMP efflux in the rat ventral hippocampus in vivo. Behav Pharmacol 5:299-305. Medline

Deacon RM, Bannerman DM, Rawlins JN (2002) Anxiolytic effects of cytotoxic hippocampal lesions in rats. Behav Neurosci 116:494-497. CrossRef Medline

Felix-Ortiz AC, Beyeler A, Seo C, Leppla CA, Wildes CP, Tye KM (2013) BLA to vHPC inputs modulate anxiety-related behaviors. Neuron 79: 658-664. CrossRef Medline

Ferguson JN, Aldag JM, Insel TR, Young LJ (2001) Oxytocin in the medial amygdala is essential for social recognition in the mouse. J Neurosci 21: 8278-8285. Medline

File SE, Pope JH (1974) Social interaction between drugged and undrugged rats. Anim Learn Behav 2:161-164. CrossRef

Gonzalez LE, Andrews N, File SE (1996) 5-HT1A and benzodiazepine receptors in the basolateral amygdala modulate anxiety in the social interaction test, but not in the elevated plus-maze. Brain Res 732:145-153. CrossRef Medline

Gradinaru V, Zhang F, Ramakrishnan C, Mattis J, Prakash R, Diester I, Goshen I, Thompson KR, Deisseroth K (2010) Molecular and cellular approaches for diversifying and extending optogenetics. Cell 141:154-165. CrossRef Medline

Guastella AJ, Einfeld SL, Gray KM, Rinehart NJ, Tonge BJ, Lambert TJ, Hickie IB (2010) Intranasal oxytocin improves emotion recognition for youth with autism spectrum disorders. Biol Psychiatry 674:692-694. CrossRef Medline

Himmler BT, Pellis VC, Pellis SM (2013) Peering into the dynamics of social interactions: measuring play fighting in rats. J Vis Exp 71:e4288. CrossRef Medline

Jonason KR, Enloe LJ (1971) Alterations in social behavior following septal and amygdaloid lesions in the rat. J Comp Physiol Psychol 75:286-301. CrossRef Medline

Kaidanovich-Beilin O, Lipina T, Vukobradovic I, Roder J, Woodgett JR (2011) Assessment of social interaction behaviors. J Vis Exp 48:2473. CrossRef Medline

Katayama T, Jodo E, Suzuki Y, Hoshino KY, Takeuchi S, Kayama Y (2009) Phencyclidine affects firing activity of basolateral amygdala neurons related to social behavior in rats. Neuroscience 159:335-343. CrossRef Medline

Killgore WD, Yurgelun-Todd DA (2005) Social anxiety predicts amygdala activation in adolescents viewing fearful faces. Neuroreport 16:16711675. CrossRef Medline

Kim JA, Szatmari P, Bryson SE, Streiner DL, Wilson FJ (2000) The prevalence of anxiety and mood problems among children with autism and Asperger syndrome. Autism 4:117. CrossRef

Kling A (1974) Differential effects of amygdalectomy in male and female nonhuman primates. Arch Sex Behav 3:129-134. CrossRef Medline

Kling A, Steklis HD (1976) A neural substrate for affiliative behavior in nonhuman primates. Brain Behav Evol 13:216-238. CrossRef Medline

Machado CJ, Emery NJ, Capitanio JP, Mason WA, Mendoza SP, Amaral DG (2008) Bilateral neurotoxic amygdala lesions in rhesus monkeys (Macaca Mulatta): consistent pattern of behavior across different social contexts. Behav Neurosci 122:251-266. CrossRef Medline

McHugh SB, Deacon RM, Rawlins JN, Bannerman DM (2004) Amygdala and ventral hippocampus contribute differentially to mechanisms of fear and anxiety. Behav Neurosci 118:63-78. CrossRef Medline

O’Donnell P, Grace AA (1995) Synaptic interactions among excitatory afferents to nucleus accumbens neurons: hippocampal gating of prefrontal cortical input. J Neurosci 15:3622-3639. Medline

Owen SF, Tuncdemir SN, Bader PL, Tirko NN, Fishell G, Tsien RW (2013) Oxytocin enhances hippocampal spike transmission by modulating fastspiking interneurons. Nature 500:458-462. CrossRef Medline

Pentkowski NS, Blanchard DC, Lever C, Litvin Y, Blanchard RJ (2006) Effects of lesions to the dorsal and ventral hippocampus on defensive behaviors in rats. Eur J Neurosci 23:2185-2196. CrossRef Medline

Petreanu L, Huber D, Sobczyk A, Svoboda K (2007) Channelrhodopsin-2assisted circuit mapping of long-range callosal projections. Nat Neurosci 10:663-668. CrossRef Medline

Pikkarainen M, Rönkkö S, Savander V, Insausti R, Pitkänen A (1999) Projections from the lateral, basal, and accessory basal nuclei of the amygdala to the hippocampal formation in rat. J Comp Neurol 403:229-260. CrossRef Medline 
Sams-Dodd F, Lipska BK, Weinberger DR (1997) Neonatal lesions of the rat ventral hippocampus result in hyperlocomotion and deficits in social behaviour in adulthood. Psychopharmacology 132:303-310. CrossRef Medline

Sanders SK, Shekhar A (1995a) Anxiolytic effects of chlordiazepoxide blocked by injection of GABAA and benzodiazepine receptor antagonists in the region of the anterior basolateral amygdala of rats. Biol Psychiatry 37:473-476. CrossRef Medline

Sanders SK, Shekhar A (1995b) Regulation of anxiety by GABAA receptors in the rat amygdala. Pharmacol Biochem Behav 52:701-706. CrossRef Medline

Schultz RT (2005) Developmental deficits in social perception in autism: the role of the amygdala and fusiform face area. Int J Dev Neurosci 23: 125-141. CrossRef Medline
Silverman JL, Yang M, Lord C, Crawley JN (2010) Behavioural phenotyping assays for mouse models of autism. Nat Rev Neurosci 11:490-502. CrossRef Medline

Tye KM, Prakash R, Kim SY, Fenno LE, Grosenick L, Zarabi H, Thompson KR, Gradinaru V, Ramakrishnan C, Deisseroth K (2011) Amygdala circuitry mediating reversible and bidirectional control of anxiety. Nature 471:358-362. CrossRef Medline

Wang DV, Wang F, Liu J, Zhang L, Wang Z, Lin L (2011) Neurons in the amygdala with response-selectivity for anxiety in two ethologically based tests. PLoS One 6:e18739. CrossRef Medline

Winslow JT (2003) Mouse social recognition and preference. In: Current protocols in neuroscience (Crawley JN, ed). Unit 8.16. 